'W Oiu, ${ }^{2} \mathrm{H}$ Shen. 'Department of Neurosurgery, Hangzhou Second Hospital, College of Medicine, Hangzhou Normal University; ${ }^{2}$ Department of Neurosurgery, Second Affiliated Hospital, College of Medicine, Zhejiang University, Hangzhou, China

Background and Aims We investigate the clinical characteristics and the microsurgery for tumors in the fourth ventricle tumors in children.

Methods The clinical data of 18 cases of pediatric fourth ventricular tumor were prospectively analyzed. The main clinical manifestations were headache ( 16 cases), vomiting ( 6 cases), visual impairment and positive Romberg sign. The imagination examination showed the tumor was in fourth ventricle, diametered from $2.5 \mathrm{~cm}$ to $7 \mathrm{~cm}$. On CT or MRI, all the patients manifested with hydrocephalus.

Results Operation was carried out under microsurgical conditions. According to the size and the position the tumor, different operation approach was performed. Median suboccipital approach was adopted for 6 cases, and cerebellomedullary fissure approach for 12 cases. The bone window was $4 \mathrm{~cm} \times 3 \mathrm{~cm}$. Total removal of the tumor was made for 16 cases, subtotal removal for 2 cases. Eight patients had external ventricular drainage during operations. The postoperative pathology finding were as follows. There were 13 cases of medulloblastoma, 2 cases of hemangioblastomas, 2 cases of ependymomas, and I case of pilocytic astrocytoma. Postoperative radiotherapy was achieved for 11 patients. The main symptoms were all improved for all patients. There were no complications and recurrence of tumors after follow-up of 2 years.

Conclusions According the size, the position and the pathological findings, the combined therapy is essential, and microsurgery is effective for tumors in the fourth ventricle tumors in children.

\section{WITCH DRUGS CHOOSE FOR SEDATION FOR COMPUTED TOMOGRAPHY IMAGING IN PEDIATRIC PATIENTS, MIDAZOLAM OR PROPOFOL?}

doi:10.1136/archdischild-2012-302724.0978

Z Addou. Anesthesie-Reanimation EHS Canastel, University of Oran, Oran, Algeria

Objective To compare safety and efficacy between propofol and midazolam as sedative agents for computed tomography Imaging (CT) in children.

Material and Methods Clinical trial prospective which included 149 children who are sedated either with propofol or midazolam over 6 months period. All children below 6 years were sedated and had I-III ASA. The level of sedation, induction time, efficacy and adverse events were recorded.

Results 66 children are sedated with midazolam (mean age: $1.8 y$ ears. sex ratio 0.8 ) and 83 children were sedated with propofol (mean age was 1.9 years; sex ratio 0,6 ). of the 149 procedures, $76 \%$ brain, 18.1 chests, and $4.7 \%$ abdomen were scanned with mean duration for midazolam $6.8 \mathrm{~min}$ and for propofol $7.3 \mathrm{~min}$ and with the mean dose for midazolam $0.31 \mathrm{mg} / \mathrm{kg}$ and for propofol $1.66 \mathrm{mg} / \mathrm{kg}$. the Ramsay sedation scale for midazolam was 2-4 and for propofol was $4-5(p<0,001)$. The induction time with midazolam was $3.2 \pm 3.8 \mathrm{~min}$ and $2.9 \pm 2.5 \mathrm{~min}$ with propofol. The completion of CT was $80 \%$ for midazolam and $95 \%$ for propofol $(p<0,001)$. Desaturation was similar between these two drugs. The intervention needed is just the administration of supplemental oxygen. The mean duration of sedation and recovery were also similar.

Conclusion Midazolam and propofol have similar efficacy and safety for computed tomography imaging imaging in children.

\section{TRANSCRANIAL DOPPLER (TCD) IN SEVERE TRAUMA BRAIN INJURIES (TBI) IN PEDIATRIC INTENSIVE CARE UNIT (PICU) IN ALGERIA. PRELIMINARY RESULTS}

doi:10.1136/archdischild-2012-302724.0979
MA Negadi, H Bouguetof, K El Halimi, D Boumendil, ZC Mentouri. Pediatric Intensive Care Unit, Faculty of Medicine - Oran University, Oran, Algeria

Background and Aims TCD is a non invasive cerebral circulation monitoring tool by evaluation of cerebral blood flow velocities in the circle of Willis.

The Aim is to show what extent the TCD allows to detect the intracranial hypertension and test the hypothesis that in children with severe TBI, there is a correlation between intracranial pressure (ICP) and TCD values.

Methods Data were prospectively collected from consecutive TCD studies in children with severe TBI undergoing ICP monitoring. For each examination of the TCD we measured the systolic, diastolic and mean velocities respectively, and we calculated the pulsatility index (PI). Middle cerebral artery through the temporal window was used for examinations of the TCD. For each patient 2-4 Doppler examinations have been recorded systematically and at ICP peaks.

Results 58 children underwent 232 TCD. There was a weak relationship between mean values of ICP and PI. Any increase in ICP above $20 \mathrm{mmHg}$ was accompanied by a PI $>1.4$ with a diastolic velocity $<30 \mathrm{~cm} / \mathrm{s}$. The CPP is comparable to the figures estimated by CPP with TCD.

Conclusion TCD has become an essential tool in the management of children for diagnosis of intracranial hypertension. Its use as in particular following traumatic brain injury, and confirmation of a clinical diagnosis of brain death by documentation of cerebral circulatory arrest. Pulsativity index (PI) and diastolic velocity $(\mathrm{Vd})$ give sufficient information to evaluate the resistance status of small downstream arteries and an indicator cerebral hypoperfusion.

\section{TRANSCRANIAL DOPPLER MONITORING IN TRAUMATIC BRAIN INJURY IN CHILDREN}

doi:10.1136/archdischild-2012-302724.0980

K Cardoso, F Vieira, F Abecasis, P Baptista, L Boto, J Rios, C Camilo, M Vieira M Correia. Paediatrics Intensive Care Unit, Hospital de Santa Maria, CHLN, Lisbon, Portugal

Background and Aims Intracranial hypertension is a serious complication of traumatic brain injury (TBI) in children and adversely affects outcome. Monitoring intracranial pressure (ICP) requires an invasive procedure. The aim of this study was to evaluate the accuracy of a noninvasive method of estimating ICP - transcranial Doppler (TCD) derived Pulsatility Index (PI) - when compared to invasive ICP measurements.

Methods Children admitted to our pediatric intensive care unit with severe TBI and ICP invasive monitoring during the study period (Aug 2008 to Mar 2012) were included in the study. TCD was done in all children and PI calculated.

Results Eighteen children met the inclusion criteria. Male:female ratio was 2.6:1. Mean age at admission was 8.7 years (14 months-17 years). Mortality rate was $11 \%$ (2/18). Mean PRISM score was 19 with a predicted mortality rate of $28 \%$. All patients except one had ICP $>20 \mathrm{mmHg}$, with a mean highest ICP of 37 $\mathrm{mmHg}(16-50)$. The first measurement of PI had a mean of 1.23 (0.55-2.95). There was a significant correlation between the first PI and corresponding ICP (Pearson correlation coefficient of 0.78 ; $\mathrm{p}<0.0001)$. When all PI were considered (41 measurements) the correlation was not significant. After excluding TCD with signs of vasospasm the correlation was again significant $(r=0.67$, $\mathrm{p}<0.001)$

Conclusions PI is a non invasive method of evaluating ICP with a strong correlation with invasive ICP measurements at admission. After a few days other factors like vasospasm must be taken into consideration when interpreting PI values. 
F Ali, R Spendiff. Radiology Department, Colchester Hospital University Foundation Trust, Colchester, UK

Background The first radiological investigation in children presenting with suspected non-accidental injury is often the skeletal survey. The purpose of a skeletal survey is to provide a standard series of radiographic images that will visualise the entire skeleton. The Royal College of Radiologists in collaboration with the Royal College of Paediatrics and Child Health has published Standards for Radiological Investigations of Suspected Non-accidental Injury. It includes imaging of ten sets of body parts. National target is $100 \%$.

Methods It was a retrospective study. Local practice was checked against National standards. All cases with suspected non-accidental injury under the age of 2 presented within last 3 years were included in this study. Case notes and radiology system was reviewed to collect data. Information was recorded on Proforma $\&$ analysed.

Results Total number of children presented during this time period were 27 . Out of which 17 were males and 10 were females. Only $18.5 \%$ had complete set of $\mathrm{x}$ rays as a part of skeletal survey, which is far less than national recommended standards. Skull, Ribs and Spine were missed in more than $30 \%$ of cases. Results were discussed locally and causes were identified. Changes were made to practice including all cases should be discussed in multidisciplinary meetings, reports counter signed by pediatric radiologist and awareness of national guidelines.

Conclusion Adherence to protocols in this context is currently poor. Non-accidental injury has medico-legal and children safety aspects. Skeletal survey is an important tool to diagnose suspects accurately which should be used wisely.

\section{MATERNAL AGE, HISTORY OF MIGRATION AND INCIDENCE OF INFANTILE HYPERTROPHIC PYLORIC STENOSIS IN GERMANY IN 2000-2008}

doi:10.1136/archdischild-2012-302724.0982

1J de Laffolie, ${ }^{2} S$ Turial, ${ }^{2} \mathrm{~F}$ Schier. 'General Pediatrics and Neonatology, University of Gießen, Gießen; '2Department of Pediatric Surgery, University of Mainz, Mainz, Germany

Background The incidence of infantile hypertrophic pyloric stenosis (IHPS) is highly variable over time and geographic regions. A decline in IHPS incidence was recently reported in Sweden, the US, Denmark, and Scotland and in Germany.

Aim In further evaluation of our previous epidemiological data, we collected data on maternal age and history of migration in mothers from the regional administrations. We examined correlations between these factors and IHPS incidence.

Methods Data were extracted from the public report of Health (Gesundheitsberichterstattung des Bundes) and population data from federal state governments. We collected the numbers of IHPS (International Statistical Classification of Diseases and Related Health Problems, 10th revision [ICD-10], code 40.0), SIDS (ICD-10, R95), and live births (LB; male/female) in each federal state for 2000-2008. Further data were collected from federal state administrations on age of mothers at birth of first child and history of migration in \% of all mothers at first birth.

Results The IHPS Incidence declined in Germany from 2000 (3.2086/1000LB [range 1.67-5.33]) to 2008 (2.0175/1000LB [1.74$3.72] ; p=0.005)$. The recorded incidence was highly variable in different federal states and over time. Negative correlation between percentage of mothers with history of migration and maternal age at first birth on the one side and IHPS incidence in the different regions and years was significant.
Conclusion The IHPS incidence declined by about 38\% nationwide. The wide variation in time and different regions is significantly correlated with maternal age and history of migration.

\section{EPIDEMIOLOGICAL PROFILE OF THE SERVICE PLAN FOR THE HIGH RISK IN MATERNAL-FETAL PUBLIC TEACHING HOSPITAL}

doi:10.1136/archdischild-2012-302724.0983

'A Veiga, ${ }^{2} \mathrm{EQ}$ Veiga, ${ }^{3} \mathrm{MCB}$ Soares, ${ }^{2} \mathrm{NV}$ Moliterno, ${ }^{2} \mathrm{SA}$ Nogueira, ${ }^{2} \mathrm{FM}$ Moliterno, ${ }^{2} \mathrm{SS}$ Cordeiro, ${ }^{4}$ Cordebel, ${ }^{4}$ A Siqueira, ${ }^{4}$ RJ Silva. ${ }^{1}$ Paediatrics and Neonatology; ${ }^{2}$ Faculdade de Medicina de Petrópolis; ${ }^{3}$ Paediatrics, Faculdade de Medicina de Petrópolis; ${ }^{4}$ Hospital Alcides Carneiro/FMP, Petrópolis, Brazil

Background and objective To know the epidemiological profile of health services maternal and child care is fundamental importance for the development of quality indicators that contribute to the quality of care for both mother and child. This study aim was to identify the epidemiological profile of the mother and the fetus seen at maternal and child public service only reference in the mountainous region of the State of Rio de Janeiro, Brazil.

Methods Cross sectional study of deliveries in the period from January to June 2011. Variables analyzed: maternal age, gestational age, parity, delivery type, sex, anthropometry and destination of the conceptus.

Results During the study period there were 781 deliveries, $45.3 \%$ of cesareanas. As the fetus to term and $65.2 \%$ frequency of gender equitable. Maternal age was 25.6 years and average parity of 2.5 with $32 \%$ primiparous pregnancies. Mean weight, height, head circumference, thoracic, abdominal and Apgar scores were 3019g, 47.5 $\mathrm{cm}, 33.6 \mathrm{~cm}, 32.5 \mathrm{~cm}, 31 \mathrm{~cm}, 8$ and 9 respectively. Of the total live births, $0.6 \%$ evolved to death in the delivery room, $12 \%$ referred to the neonatal ICU and of these $61.57 \%$ for prematurity.

Conclusion We are facing a referenced service to high-risk pregnancy and cesarean rates of prematurity are above the level recommended by the Ministry of Health as an ideal, what leads us to reflect on the need for planning actions to be implemented with goal of greater control and quality of care offered to this same population.

\section{POPULATION-BASED STUDY TO EVALUATE THE NEONATAL MORBIDITY AND MORTALITY IN MATERNAL DIABETES}

doi:10.1136/archdischild-2012-302724.0984

${ }^{1} \mathrm{~A}$ Lange, ${ }^{2 \mathrm{R}}$ Spoo, ${ }^{3 \mathrm{R}}$ Thyrian, ${ }^{3} \mathrm{~W}$ Hoffmann, ${ }^{4} \mathrm{H}$ Lode, ${ }^{2} \mathrm{M}$ Heckmann. ${ }^{\top}$ Neonatology and Intensiv Care, University of Greifswald; ${ }^{2}$ Neonatology and Pediatric Intensive Care, University Children's Hospital; ${ }^{3}$ Community Medicine, University of Greifswald ${ }^{4}$ Haematology and Oncology, University Children's Hospital, Greifswald, Germany

Aim The aim of the study is to determine any connections between maternal diabetes and the pregnancy outcome of mothers and newborns.

Methods From 03/2003 to $11 / 2008$ in local and university hospitals in Northeast Pomerania, Germany a total of $n=4593$ mothers and their children were used as a prospective population-based birth cohort. The objective of the survey of newborns in Pomerania ( $\mathrm{SNiP}$ ) is to evaluate the negative pregnancy outcome, morbidity and mortality in diabetes affected pregnant woman. A comparison of glucose-intolerance affected childbearing mothers $(n=243)$ identified by oral glucose tolerance test and non-diabetic mothers $(\mathrm{n}=4350)$ were conducted.

Results The prevalence of maternal glucose-intolerance is $5.3 \%$, Comorbidity of maternal diabetes is more prevelant with higher age, greater number of previous pregnancies (parity) and those who are overweight (relative risk 1.97). Macrosomi $(\mathrm{OR}=2.28)$ and reduced gestational age are identified as a fetal risk factors. As a maternal risk factor is considered positive vaginal infection $(O R=1.97)$. As a 